MATEC Web of Conferences 4, 01002 (2013)

DOI: $10.1051 /$ matecconf/20130401002

(C) Owned by the authors, published by EDP Sciences, 2013

\title{
Anthracene and TCNQ doping of substituted nickel phthalocyanine: Effects on the electrical and optical properties of spin coated thin films
}

\author{
Aseel K. Hassan ${ }^{1 *}$, Tamara Basova², Ayse Gül Gürek ${ }^{3}$, Vefa Ahsen ${ }^{3,4}$ \\ ${ }^{1}$ Materials and Engineering Research Institute, Sheffield Hallam University, City Campus, Sheffield S1 1WB, UK \\ email address; a.hassan@,shu.ac.uk \\ ${ }^{2}$ Nikolaev Institute of Inorganic Chemistry, Lavrentiev Ave., 3, Novosibirsk 630090, Russia \\ ${ }^{3}$ Gebze Institute of Technology, Department of Chemistry, Gebze, Kocaeli, Turkey \\ ${ }^{4}$ TUBITAK-Marmara Research Center, Materials Institute, Gebze, Kocaeli, Turkey
}

\begin{abstract}
In this work, we study the controlled doping of thin films of substituted nickel phthalocyanine, $\mathrm{NiPcR}_{8}$, where $\mathrm{R}=-\mathrm{SC}_{6} \mathrm{H}_{13}$, with anthracene and TCNQ and investigate the effects of the extent of this doping on the optical and electrical properties of $\mathrm{NiPcR}_{8}$ films. Optical constants, namely index of refraction (n) and extinction coefficient $(\mathrm{k})$ have increased for both types of doping, as determined from spectroscopic ellipsometry measurements. Conductivity is shown to increase by about four orders of magnitudes compared to pure $\mathrm{NiPcR}_{8}$ films as a result of anthracene doping while TCNQ has resulted in almost six orders of magnitudes increase in the film's conductivity.
\end{abstract}

\section{Introduction}

Organic semiconductors are promising materials for photovoltaic cells and other optoelectronic applications such as light emitting diodes (LEDs) and optical switches. Several groups have shown that the photovoltaic performance of organic-based devices can be enhanced by doping the organic material with oxidizing gases or liquids like iodine or bromine [1, 2].

An improvement of both the photovoltage and the photocurrent was achieved for low-doping concentrations. Such organic semiconductors as tetracyanoquinodimethane (TCNQ) and anthracene are also used as doping agents [3]. A systematic study on doping of vanadyl and zinc phthalocyanines by a fully fluorinated form of tetracyanoquinodimethane as an example of controlled doping of thin organic films by cosublimation of matrix and dopant was carried out [4]. Anthracene doped with metal-free phthalocyanine was prepared by the co-crystallization method and its activation energy is determined [5]. The unique columnar architecture of discotic liquid crystals (DLCs) combined with their self-organising properties and the ease with which they form bulk-aligned samples makes them attractive for a range of applications as 'one-dimensional' conductors $[6,7]$.

It is known that the electrical conduction in liquid crystals and in particular in phthalocyanine-based materials is greatly affected by contaminants and environmental conditions [7]. The problem of time and temperature instability of the electrical current related to parasitic doping effects in discotic liquid crystals was studied in Ref. [8] using metal-free mesogenic 2(3),9(10),16(17),23(24)-tetra(2-decyltetradecyloxy) phthalocyanine as an example. The influence of a controlled doping of the films of LC phthalocyanines is not studied in detail.

In this work, we study the controlled doping of thin films of substituted nickel phthalocyanine, $\mathrm{NiPcR}_{8}$, where $\mathrm{R}=-\mathrm{SC}_{6} \mathrm{H}_{13}$, with anthracene and TCNQ and investigate the effects of the extent of this doping on the optical and electrical properties of $\mathrm{NiPcR}_{8}$ films.

\section{Materials and Methods}

$\mathrm{NiPcR}_{8}$ (see Fig. 1) was synthesized as described in Ref. [9].

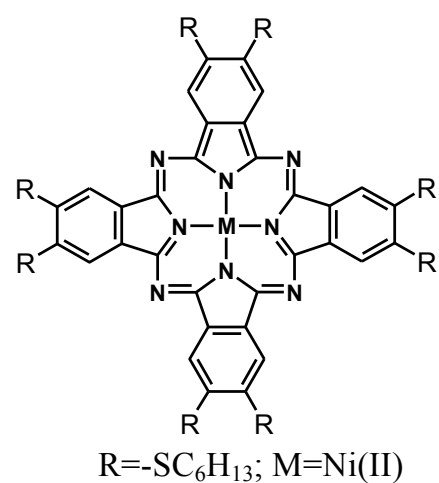

Fig. 1: Molecular structure of $\mathrm{NiPcR}_{8}$ 
A small volume of the solution of $\mathrm{NiPcR}_{8}(5 \mathrm{mg} / \mathrm{ml})$ with added TCNQ or anthracene (1 weight \%) in chloroform was dispensed via a glass pipette onto an ultrasonically glass substrate held onto photoresist spinner (Microsystem model 4000). The substrate was spun at $2000 \mathrm{rpm}$ for $30 \mathrm{~s}$ and the solvent had evaporated during this period, producing a homogeneous film. The substrates used were varied according to experimental requirements for different characterisation techniques.

The resulting films of $\mathrm{NiPcR}_{8}$ were heated at the temperature of $150{ }^{\circ} \mathrm{C}$ during 6 hours in order to obtain ordered films due to their liquid crystalline properties as described in [10]. Spectroscopic ellipsometric measurements on $\mathrm{NiPcR}_{8}$ films spun on silicon substrates were performed by using a Woolam $M-2000 V^{T M}$ rotating analyser spectroscopic ellipsometer in the spectral range of 370-1000 nm. Atomic force microscopy (AFM) was obtained from Nanoscope 1lla Multimode Atomic microscope. Current-voltage measurements were performed using a Keithley 6517A electrometer equipped with a microprocessor controlled measuring system. For the measurements of the in-plane film conductivity, the films were spun onto glass substrates with interdigitated electrodes (IDE) at an interelectrode distance $\mathrm{L}=60 \mu \mathrm{m}$ and at a width of electrode overlap $\mathrm{W}=3.125 \mathrm{~mm}$. All electrical measurements were performed in air at room temperature.

\section{Results and Discussion}

Spectroscopic ellipsometry was used for the characterization of thickness, refractive index (n) and extinction coefficient $(\mathrm{k})$ of the $\mathrm{NiPcR}_{8}$ films deposited on silicon substrates. Using Levenberg-Marquardt multivariate regression algorithm, the measured ellipsometric data were fitted to the Cauchy model:

$$
\begin{aligned}
& n(\lambda)=A+\frac{B}{\lambda^{2}}+\frac{C}{\lambda^{4}} \\
& k(\lambda)=\beta \exp \left[\gamma\left(\frac{1}{\lambda}-\frac{1}{L}\right)\right]
\end{aligned}
$$

where $\mathrm{n}$ and $\mathrm{k}$ are the index of refraction and extinction coefficient at wavelength $\lambda$ and $\mathrm{A}, \mathrm{B}, \mathrm{C}, \beta, \gamma$ and $\mathrm{L}$ are model parameters. A detailed description of the principle of ellipsometry can be found in Ref. [11].

Resulting variation of refractive index and extinction coefficient of pure $\mathrm{NiPcR}$ films and the films of $\mathrm{NiPcR}_{8}$ doped with anthracene and TCNQ deposited at 2000 r.p.m. with incident photon wavelength are shown in Fig. 2 .

The estimated thickness and optical constants measured at $\lambda=633 \mathrm{~nm}$ are given in Table 1 . The index of refraction (n) has increased for both types of doping, as determined from spectroscopic ellipsometry measurements. The thickness of the pure $\mathrm{NiPcR}_{8}$ film was $72 \mathrm{~nm}$, the thicknesses of the films dopped with anthracene and TCNQ were 74 and $78 \mathrm{~nm}$, respectively.

Atomic force microscopy has been used to study the effect of doping on film morphology.
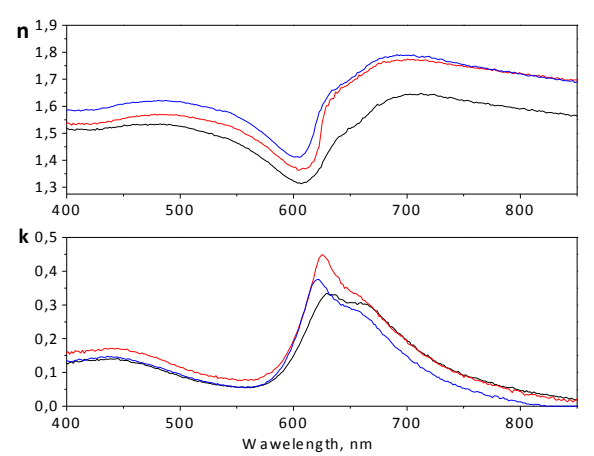

Fig. 2. $\mathrm{n}$ and $\mathrm{k}$ for pure (black line), anthracene (red line) and TCNQ doped (blue line) $\mathrm{NiPcR}_{8}$ films.

Table 1: Optical of constants of pure, Anthracene and TCNQ doped $\mathrm{NiPcR}_{8}$ films.

\begin{tabular}{|c|c|c|c|}
\hline Film & Thickness (nm) & $\mathrm{n}$ & $\mathrm{k}$ \\
\hline $\mathrm{NiPcR}_{8}$ (Pure) & 786 & 1.53 & 0.36 \\
\hline $\begin{array}{c}\text { NiPcR } \\
\text { (antracene doped) }\end{array}$ & 722 & 1.63 & 0.41 \\
\hline $\begin{array}{c}\text { NiPcR } \\
\text { (TCNQ doped) }\end{array}$ & 741 & 1.66 & 0.33 \\
\hline
\end{tabular}

Microstructures obtained by AFM (Fig. 3) show that spun films of pure $\mathrm{NiPcR}_{8}$ exhibit more surface roughness characteristic with smaller grain size compared to the films of $\mathrm{NiPcR}_{8}$ doped with anthracene and TCNQ. Increased doping concentration is shown to lead to increased film roughness probably due to precipitation of the doping molecules in the film. The relatively large scale used to obtain AFM images of films studied in the present work may not allow us to make clear speculation about films' crystallinity. However, an X-ray diffraction pattern of a similar system comprising perylene doped copper phthalocyanine films has shown that increased perylene doping resulted in reduced film's crytallinity [12].
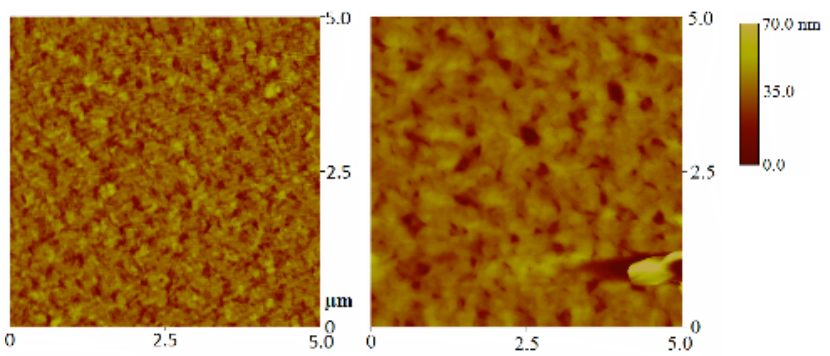

Fig. 3. AFM images for the pure and TCNQ doped $\mathrm{NiPcR}_{8}$ films.

The latter was supported by SEM observations, which showed that the film's surface became rough as a result of increased dopant concentration and was ascribed to perylene precipitation. Figure 4 shows the DC electrical characteristics plotted in log-log scales for the pure $\mathrm{NiPcR}_{8}$ film (curve a), anthracene-doped $\mathrm{NiPcR}_{8}$ film 
(curve b) and TCNQ-doped $\mathrm{NiPcR}_{8}$ film, all spin-casted onto ITO-coated glass slides and coated with thermally evaporated Al contacts produced under vacuum of $10^{-5}$ mbar. It is clearly shown that anthracene doping has increased the conductivity by about four orders of magnitudes compared to pure $\mathrm{NiPcR}_{8}$ films while TCNQ has resulted in almost six orders of magnitude increase in the film's conductivity. This increase in conductivity could be ascribed to an increase in hole concentration as a result of Fermi level position shift towards valence band edge which is caused by the anthracene and TCNQ impurities. Similar effects were observed for other phtahlocynaines films when doped with oxygen [13] and iodine [2]. Iodine doping was also shown to enhance the photoresponse of $\mathrm{ZnPc}$ thin films as well as improving diode parameters of an ITO/ZnPc/Al diode structure [2]. Similarly iodine doping of thin films of Ndocosylpyridinium-tetracyanoquinodimethane (TCNQ) salts prepared by Langmuir-Blodgett (LB) method has caused an extensive improvement to the TCNQ films' conductivity $[14,15]$.

Figure 4 also shows that all three device structures exhibit ohmic conduction at low voltages $(\mathrm{V} \leq 0.4 \mathrm{~V})$ with a gradient of $\sim 1$ and thus satisfying the following relation:

\section{$I \propto V / d$}

where $\mathrm{d}$ is the film thickness. At applied voltages larger than $0.4 \mathrm{~V}$, electron transport may be ascribed to trapcontrolled conduction where the gradients of $\log (\mathrm{I})$ versus $\log (\mathrm{V})$ in all three studied samples have displayed a value of $\sim 2$, which may suggest space charge limited conduction (SCLC) [16]. This type of conduction can only be confirmed when such a square-law dependence $\left(I \propto V^{2}\right)$ extends over a sufficient range of applied voltage, and the measured current satisfies film thickness dependence as described by the following formula:

$$
I \propto V^{2} / d^{3}
$$

Such type of conduction mechanism may be attributed to space charge limited conduction (SCLC) controlled by a single dominating trap level [17].

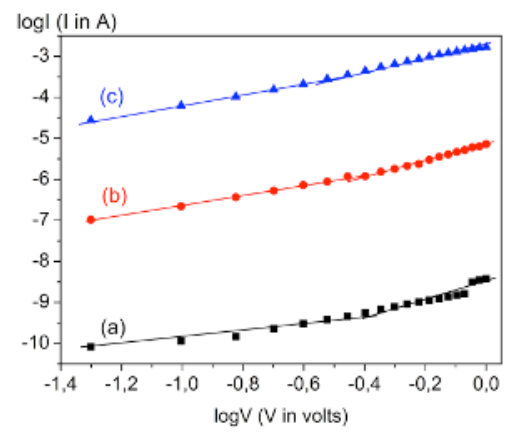

Fig. 4. DC current as a function of applied voltage for pure $\mathrm{NiPcR}_{8}$ (a) anthracene-doped $\mathrm{NiPcR}_{8}$ (b) and TCNQ-doped $\mathrm{NiPcR}_{8}(\mathrm{c})$ films.

In Fig. 5 AC conductance as a function of frequency $(\mathrm{G}(\omega))$ is shown to satisfy the relation $\mathrm{G}(\omega)=\mathrm{A} \omega^{\mathrm{s}}$ where the power $\mathrm{s}$ is a function of both frequency and temperature. The magnitude of index $\mathrm{s}$ provides information on the type of electronic conduction mechanism over the different regimes of frequency and temperatures. In the present work $\mathrm{G}(\omega)$ is shown to be frequency independent for both doped films yielding a value of $\mathrm{s}=0$ up to $\mathrm{f}=30 \mathrm{kHz}$ and then increases to unity beyond $30 \mathrm{kHz}$.

In pure $\mathrm{NiPcR}_{8}$ films on the other hand $\mathrm{AC}$ conductance is shown to exhibit strong frequency dependence with the index s exceeding unity. It can therefore be inferred here that electronic conduction in doped films is mainly due to band conduction, especially at lower frequencies while electronic conduction in pure $\mathrm{NiPcR}_{8}$ films is mainly due to hopping of charge carrier.

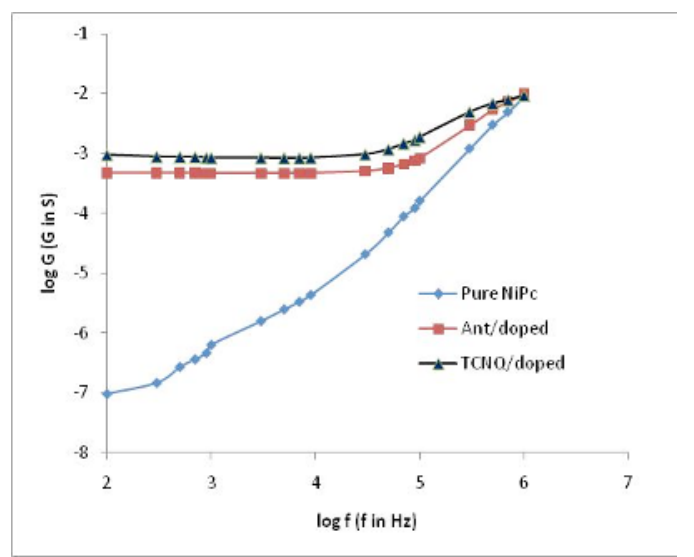

Figure 5: AC conductance as a function of frequency. for pure NiPcR8, anthracene-doped, and TCNQ-doped NiPcR 8 films.

\section{Conclusion}

Effects of anthracene and TCNQ doping on the structural, optical and electrical properties of substituted nickel phthalocyanine thin films have been investigated. AFM images show that pure $\mathrm{Ni}\left(\mathrm{PcR}_{8}\right)_{2}$ films exhibit more crystalline characteristics with smaller grain size compared to anthracene and TCNQ doped films. Increased doping concentration leads to increased film roughness probably due to precipitation of the doping molecules in the film. Films' conductivity increased by nearly six orders of magnitude as a result of TCNQ doping and DC conduction at high applied fields was primarily ascribed to trap-controlled space charge limited conduction, with traps homogeneously distributed in energy within the band gap.

\section{References}

1. W.A. Nevin, G.A. Chamberlain, J. Appl. Phys. 69, 4324 (1991).

2. G.D. Sharma, S.G. Sangodkar, M.S. Roy, Mater. Sci. Eng. B 41, 222 (1996).

3. J.V. Joseph, F.P. Xavier, Bull. Mater. Sci. 21, 1 (1997).

4. M. Pfeiffer, A. Beyer, B. Plönnigs, A. Nollau, T. Fritz, K. Leo, D. Schlettwein, S. Hiller, D. Wöhrle, Solar Energy Materials \& Solar Cells 63, 83 (2000). 
5. S. Lawrence Selvaraj, Francis P. Xavier, J. Cryst. Growth 233, 583 (2001).

6. M.G. Debije, J. Piris, M.P. de Haas, J.M. Warman, Z. Tomovic, C.D. Simpson, M.D. Watson, K. Müllen, J. Am. Chem. Soc. 126, 4641 (2004).

7. F.I. Bohrer, A. Sharoni, C. Colesniuc, J. Park, I.K. Schuller, A.C. Kummel, W.C. Trogler, J. Am. Chem. Soc. 129, 5640 (2007).

8. A. Calò, P. Stoliar, M. Cavallini, Y.H. Geerts, F. Biscarini, Organic Electronics 12, 851 (2011).

9. A.G. Gürek, Ö. Bekaroğlu, J. Chem. Soc. Dalton Trans., 1419 (1994).

10. T.V. Basova, B.A. Kolesov, A.G. Gürek, V. Ahsen Thin Solid Films, 385, 246 (2001).

11. H. Arwin, D.E. Aspnes, Thin solid Films 138, 195 (1986).

12. Rudiono, F. Kaneko, M. Takeuchi, Appl. Surf. Sci., 142, 598 (1999).

13. B. Boudjema, G. Guillaud, M. Gamoudi, M. Maitrot, J.J.Andre, M. Martin and J. Simon, J. Appl. Phys. 56, 2323 (1984).

14. A. Barraud,, P. Lesieur, J. Richard, A. Ruaudel Teixier and M. Vandevyver, Thin Solid Films 133, 125 (1985).

15. M. Matsumoto, T. Nakamura, E. Manda, and Y. Kawabata, Thin Solid Films 169, 61 (1988).

16. P. D. Hooper, M. I. Newton, G. McHale and M. R. Willis, Semicond. Sci. Technol. 12, 455 (1997).

17. K.C. Kao and W. Hwang, "Electrical Transport in Solids" 1981, Oxford: Pergamon. 